Pedagogía y Saberes n. ${ }^{\circ} 54$

Universidad Pedagógica Nacional

Facultad de Educación. 2021. pp. 171-185

\title{
El campesinado en la educación rural: un debate emergente
}

The Peasantry in Rural Education: An Emerging Debate

O campesinato na educação rural, um debate emergente

Jairo Arias-Gaviria**

\section{Para citar este artículo}

Arias-Gaviria, J. (2021). El campesinado en la educación rural: un debate emergente. Pedagogía y Saberes, (54). https://doi.org/10.17227/pys.num54-10555

* El presente artículo de investigación se deriva de la tesis de maestría Educación Rural y Saberes Campesinos en Tierradentro Cauca: Caso de la Asociación Campesina de Inzá Tierradentro ACIT.

** Magíster en Educación. Docente Universidad Pedagógica Nacional, Bogotá, Colombia. Correo: jariasg@pedagogica.edu.co

Código Orcid: https://orcid.org/0000-0002-6327-9710 


\section{Resumen}

Este artículo propone una reflexión sobre la pertinencia en la ruralidad de una propuesta educativa que incluya al campesinado con sus prácticas culturales y saberes, y lo reconozca como sujeto de derechos. Los significados de ruralidad y educación rural presentes en la política nacional de educación rural aún obvian la construcción de un modelo educativo que integre categorías que claramente reconozcan a este sector como centro de aporte sociocultural y que esto se refleje en programas y mallas curriculares. Además, todavía no se logra superar la idea simplificada de la producción agrícola como factor de identificación del campesinado. Surge entonces el interrogante sobre qué condiciones impiden que dichas prácticas de esta población ingresen a los currículos escolares. En este acercamiento se presentan diferentes momentos de reflexión, asociados a pesquisas sobre los discursos acerca de la educación rural, las prácticas y vinculaciones de sectores campesinos que han propiciado iniciativas sobre el tema, en los cuales la perspectiva curricular para su reconocimiento es también el centro de discusión para su postulación como sujetos garantes de derechos. Esto concreta la síntesis sobre la necesidad de construir planes, programas y proyectos de educación que vinculen las prácticas culturales de los campesinos del país desde los primeros años de edad a la política educativa nacional con una clara perspectiva de pedagogía de lo rural.

\section{Palabras clave}

ambiente rural; reconocimiento; escuela; medio sociocultural

\section{Abstract}

This article postulates a reflection focused on the revision and relevance in rurality of an educational proposal that includes the peasantry with their cultural practices, knowledge and recognition as subjects of rights. The meanings of rurality and rural education present in the National Rural Education Policy still obviate the construction of an educational model that integrates categories where it clearly recognizes this sector as center of socio-cultural contribution reflected in curricular programs and syllabi. Furthermore, the simplified idea of agricultural production as a factor of identification of peasantry has not yet been overcome. The question then arises as to what conditions prevent such practices from this population from entering the school curricula. In this approach, different moments of reflection are undertaken, associated with research on the discourses of rural education, practices and links of peasant sectors that have fostered initiatives on the subject of rural education, where the curricular perspective for its recognition is also the discussion center for their postulation as guarantors of rights. This encompasses the synthesis of the need to build education plans, programs and projects that link the cultural practices of the peasants of the country from the first years of age to the national educational policy with a clear perspective of rural pedagogy.

\section{Keywords}

rural environment; recognition; school; sociocultural environment

\section{Resumo}

Este artigo postula uma reflexão focada na revisão e relevância na ruralidade de uma proposta educacional que inclua o campesinato com suas práticas e saberes culturais, e os reconheça como sujeitos de direitos. Os significados de ruralidade e educação rural presentes na Política Nacional de Educação Rural ainda evitam a construção de um modelo educacional que integre categorias em que reconheça claramente esse setor como centro de contribuição sociocultural refletido em programas e malhas curriculares. Além disso, a ideia simplificada da produção agrícola como fator de identificação do campesinato ainda não foi superada. Surge então a questão de quais condições impedem que essas práticas desta população entrem nos currículos escolares. Nessa abordagem, são realizados diferentes momentos de reflexão, associados à pesquisa sobre os discursos da educação rural, práticas e vínculos dos setores camponeses que fomentaram iniciativas sobre a educação rural, onde a perspectiva curricular para seu reconhecimento é também o centro de discussão para a sua postulação como sujeitos garantes de direitos. Isso abrange a síntese da necessidade de construir planos, programas e projetos educacionais que vinculem as práticas culturais dos camponeses do país desde os primeiros anos de idade à política educacional nacional com uma perspectiva clara da pedagogia rural.

\section{Palavras-chave}

ambiente rural; reconhecimento; escola; ambiente sociocultural 


\section{Introducción}

El campesinado colombiano es valorado como un sector sin importancia para la economía nacional, al tiempo que la reflexión sobre los saberes campesinos inmersos en los procesos de aprendizaje en el mundo rural para elaborar textos y ajustes al modelo educativo rural es vital para su reconocimiento. También es relevante problematizar la existencia de una identidad como campesinos y campesinas ceñidos al papel que representa la pertenencia a un territorio y su relación con la educación.

Las estrategias y los programas educativos en Colombia no han ofrecido diferencias regionales ni locales que puedan dar cuenta de su gran diversidad como campesinos, quizá, porque la noción de espacio que se concibe no refleja lo que Lefebvre (1974) describe como una construcción, y más bien conduce a una realidad de pensamiento contemporáneo que se sustenta sobre la realidad de unas lógicas sistemáticas y taxativas que llevan a tener una sola manera de dar cuenta de la realidad y comprenderla. Por ello, una educación pertinente es, además, una que pueda adaptarse a dicha diversidad; a los niños y niñas, a las comunidades indígenas, a los habitantes rurales y sus particularidades. De la misma forma, es oportuno hacer referencia a la ruralidad, a la vida y a los ritmos del campo, en la cual los campesinos sean más que una apreciación paisajística.

La aceptabilidad de la educación supone garantías de calidad, estándares mínimos de salud, seguridad y requisitos profesionales para los maestros; ello debe ser estipulado, verificado y controlado por el Gobierno. La admisibilidad de la enseñanza se ha extendido considerablemente gracias al desarrollo del derecho internacional, pero no ha sido en ningún sentido pertinente. No solo se trata de asistir a la escuela para aprender contenidos, como lo describe Arias:

también está a la base el supuesto de que ello debe responder a la vida colectiva del campo, en la que se pueda relacionar con el medio, con las plantas, con el trabajo agrícola, con la huerta, con la lluvia y las fases de la luna; ahora bien, qué elementos del medio se integran a las prácticas del docente, cómo se han constituido los programas educativos y las mallas curriculares. Una pedagogía que hable de las vías, de los caminos, de la diferencia en tonalidades del verde de las montañas, de los problemas en salud, de la minería, la felicidad, de la ternura y el sentir como moradores del campo. (2017, p. 61)

Acá la pregunta de rigor es, ¿qué tanto de esos ideales se refleja en las prácticas escolares? Si bien la sociedad se interroga por la función que cumple la escuela, las transformaciones en los currículos acaecen muchas veces sin cambios. En este contexto, las organizaciones sociales, rurales y de base buscan y proponen transformar, desde su empoderamiento, estrategia para la construcción de nación, "o nacionalidad" como lo presenta Herrera, Pinilla y Sauza (2003), con sugerencias y reclamos por una educación de "calidad" o pertinente a sus ritmos de vida con una praxis cotidiana de la ruralidad, dado que las políticas nacionales en educación no corresponden de forma adecuada a sus ritmos y necesidades.

Comprender la ruralidad en la dinámica de la educación implica valorar la vida de los pobladores rurales como acción y transformación para la vida del campo. Si bien Bourdieu (1998) y Bernstein (1972) coinciden en determinar audazmente los elementos que destacan el papel de la educación y sus fuertes contraflujos como auspiciadora de control social y poder en las formas de la conciencia, vale la pena intentar aproximarse a una mirada inquietante por la ruralidad — como lucha y criterio de análisis - y, al mismo tiempo, emprender en la educación otra forma de leer, escribir y acercar ese mundo local -en lugar de disgregar-, de narrar las imágenes orales y sentir las prácticas de un pueblo históricamente excluido que en los últimos años ha recobrado esbozos de dignidad para su reivindicación como sujetos de derechos.

La educación rural y la ruralidad reclaman cambios profundos en el campo educativo formal, porque este no responde a la dinámica ni al ritmo de los habitantes del campo, sustenta una educación de baja "calidad" o tiene bajo impacto en la construcción y la existencia de otras maneras del aprender, del hacer y del enseñar. La escuela rural debería integrar la inquietud por la integración de los saberes campesinos como proyecto educativo rural, y, en concordancia con Lefebvre (1974) nutra esa verdad del espacio que se une a la práctica social y al uso que se le da a la misma. De esta manera, toda acción de identidad educativa correspondería no solo a lo percibido del entorno rural, sino, además, a lo vivido; por tanto, la concepción educativa de lo rural implicaría también una representación del espacio.

El campesinado de Colombia hace parte del $32 \%$ de la población que habita la ruralidad según el informe de desarrollo humano elaborado en el 2011 por el Programa de las Naciones Unidas para el Desarrollo (PNUD). Al mismo tiempo, existe la contradicción por no disponer de vinculación al sistema de medición por parte del Departamento Administrativo Nacional de Estadística (Dane) en el 2018, para incluir la pregunta sobre el campesinado en el Censo Nacional de Población y Vivienda, hecho que 
ayudaría a saber con precisión cuántas personas se autodenominan campesinas en el país. Por ello, al no tener sistemas de representación diferenciable, sus connotaciones y definiciones han sido divergentes. El reto de establecer un escenario para esbozar estrategias que identifiquen procesos educativos que los reconozca es una buena estrategia de registro de su posible autodeterminación.

La educación, con todas las críticas que pueda recibir por estructurarse más con el sistema de producción mercantil, también es el nicho para auspiciar sistemas de representación y forjar identidad colectiva, como estipula Giménez (2012), en su devenir de prácticas sociales, donde construyen estructuras de sistemas simbólicos. Para el caso de los campesinos, tales estructuras pueden ser el punto de origen de pedagogías de lo rural que enuncien no solamente un reconocimiento de los campesinos como sujetos de derechos, sino que además ofrezcan una perspectiva de la vida rural de una población con prácticas culturales instauradas que bien se pueden enunciar como sistemas de saberes campesinos. Ya el pasado mes de marzo del 2020 se conoció la primera encuesta de cultura política desarrollada por el Dane, que incluye la pregunta por el campesinado. Esta arroja evidencias - aún incipientes - sobre lo que se conoce sobre este grupo en relación a las dinámicas de integración concretas como, por ejemplo, cuántas personas se consideran campesinas y cuál es su grado escolar. Si bien esta información es valiosa, deja preguntas sobre lo que la academia y la misma historicidad supone conocer de una población, pero que, en la vida cotidiana más actual, aún sigue siendo un reto por descubrir.

El presente artículo busca poner de manifiesto la relación entre campesinado y educación rural, para ello destaca elementos sobre su conceptualización como campesino en el marco de las estrategias de educción rural en el país. Luego de ello, se expone la idea de pedagogía rural dentro del proceso organizativo campesino como motor de recientes iniciativas para su reconocimiento como sujeto político, y termina con la disertación sobre la identidad campesina como axioma complejo, no solo de la educación rural, sino de su presencia al interior de programas y proyectos educativos que lo reconozca como actor de saberes y relación histórica cultural de la educación rural.

\section{Entre lo deseable y lo sensible}

En Latinoamérica ha sido notoria la dificultad para definir conceptualmente al campesino. Los inconvenientes, como reitera Hernández (1994), pasan por la no concreción de una única unidad de criterio, además, de las diversas formas y tendencias para dar cuenta de un sujeto con sus rasgos definidos. Según Ortiz, la palabra "campesino está llena de asociaciones emotivas. Pero no existe (hoy día) otra palabra que describa a los habitantes rurales que, carente de una fuerte identidad tribal, siguen marginados del mundo de las ciudades y sin embargo dependen de él" (1979, p. 288). Para el caso colombiano, la invisibilización del sector rural y sus habitantes ha integrado, como lo describe Pérez,

un sistema de crisis generalizada: crisis de la producción, crisis de la población y poblamiento, crisis de las formas de gestión tradicional, crisis en el manejo de recursos ambientales, crisis de las formas tradicionales de articulación social, así, todo el modelo de sociedad rural está en crisis. (2001, p. 21)

Al abordar la tipología rural y la pedagogía de saberes campesinos nos inscribimos en una especie de "disputa de significados", como lo menciona Apple (1997, p. 12), porque no se trata solo de evidenciar en la escuela la presencia o no de un currículo contextualizado, sino también de encarnar la lucha de pobladores que se sienten marginados, y la marginalidad también está en el contexto escolar.

De esta manera existe un escenario de la enseñanza que recuerda que "lo pedagógico y lo escolar, son dimensiones culturales y formativas [...] donde la enseñanza del currículo está inevitablemente comprometida [...] en la producción y reproducción de divisiones sociales que pasan por el modelo de identidades sociales particulares" (Tadeu da Silva, 1997, p. 72). Es posible inferir que al construir una propuesta educativa que recoja las dimensiones de la vida rural, es necesario incorporar saberes y prácticas de reconocimiento identitario de dichas poblaciones. No obstante, como lo recuerdan Martínez et ál., el diseño del plan colombiano de estudios se centró en la planificación como lo más relevante del currículo, no en las destrezas o habilidades para el aprendizaje: "planificar era lo importante, ahora se trababa de cumplir objetivos, contenidos, actividades y más tarde la evaluación" (1994, p. 55). Aunque estas nuevas formas no transformaron la escuela, sí cambiaron sus procesos de enseñanza. "Ahora el currículo aparece junto a los discursos de las prácticas del desarrollo y la planificación, tal como ocurre en los países desarrollados e industriales" (Martínez et ál., 1994, p. 50).

Claro está que esa manifestación no es únicamente moderna. Quiceno (2003) nos recuerda cómo también en la educación de la Gran Colombia el objeto de la instrucción pública era instruir al hombre individual por medio de la instrucción estatal. Esta educación tenía varios componentes: 
Para la comunicación se pensó en escuelas y algunos colegios para el pueblo, y las universidades y colegios provinciales para los grupos de poder. Por fuera de la ley se creó otras formas de educación llamadas asociaciones de individuos, conformadas por personalidades del poder económico y político. Las escuelas debían encargarse de la enseñanza de algunas nociones tales como las normas, los castigos y la transmisión de algunas ideas republicanas, los colegios se encargarían de dar paso a algunos pocos a la Universidad y por ende de algunos de ellos a la capital y su cultura. (Quiceno, 2003, p. 39)

De esta manera, la instrucción pública era únicamente para unos pocos y, por extensión, también el acceso al poder, y, "las regiones dotaban en tanto pocos elementos de educación" (p. 40). En contraposición a ello, en las asociaciones, el saber era secreto; allí sí circulaba el acceso a la ciencia, a la cultura y al poder. De esta manera,

lo que está a la base en esta época era el entramado de entender educación como parte del orden que tomaba la configuración territorial de la Gran Colombia, como producto de sus dos constituciones, la de 1821 y la de 1827 para lo cual queda instaurado una representación de poder central, desde lo alto quedaría organizado en primer lugar la teología, el derecho y la medicina, seguido de un espacio para las universidades y la capital. Hacia los lados las provincias y las regiones (colegios y escuelas) y en lo bajo el pueblo y la masa ignorante. (Quiceno, 2003, p. 43)

Ese tipo de estrategias derivaron en discursos donde la "calidad" de la educación quedó en entredicho, no por falta de propuestas de ampliación en cobertura, como lo demuestra Perfetti,

hasta los años 90s, tanto las escuelas y estudiantes rurales, han mostrado avances importantes, los cuales se deben principalmente a la expansión y consolidación de modelos educativos concebidos para estas zonas, como el caso de Escuela Nueva, el Sistema de Aprendizaje Tutorial-SAT... (2003, p. 167)

La situación socioeconómica no corrió con la misma suerte. La extrema pobreza de los habitantes rurales hizo entender mejor la condición y el desempeño de la educación en dichas zonas. Según el informe presentado por Perfetti,

el crecimiento continuo del nivel de pobreza rural durante los noventa, el cual fue del $83 \%$ hacia fines de la década, con un incremento de 10 puntos porcentuales en relación con su contraparte urbana, indica la magnitud del crecimiento de la pobreza en las zonas rurales del país. (2003, p. 172)
Las zonas rurales presentaban condiciones laborales desfavorables, desempleo, familia a temprana edad, analfabetismo, entre otros aspectos. Esto determinó un patrón de poca permanencia de la población en edad escolar en el sistema educativo. Según el informe de Perfetti,

de 100 estudiantes que se matriculan en primero de primaria en las zonas rurales, solo 35 terminan este ciclo y un poco menos de la mitad (16 estudiantes) pasan a secundaria; de estos, 8 completan el noveno grado y solo 7 culminan el ciclo completo de educación básica. (2003, p. 183)

Lo que uno se preguntaría es: ¿Qué hacen esos jóvenes que no terminan? ¿Qué tipo de actividades emprenden?

Ahora bien, desde el año de 1996 el Gobierno nacional amplió sus objetivos de fortalecimiento del Programa de Educación Rural (PER) con la intención de aumentar la cobertura y mejorar su calidad; instauró una institucionalidad con un sistema financiero que lo apoye y mantenga. Es posible que hoy día, las carencias en educación rural mantengan su índice de gravedad.

En el mismo sentido, la educación para las zonas rurales ha contado, desde la década de los cincuenta, con una serie de programas educativos que han mejorado la cobertura y han brindado oportunidades para acceder a la educación a población en extraedad y adulta, así como asistencia para mejorar el índice de analfabetismo e integración social. Debe reconocerse la importancia y presencia por parte del Ministerio de Educación Nacional (MEN) en programas como Escuela Nueva, Posprimaria Rural, Sistema de Aprendizaje Tutorial (SAT), Programa de Educación Continuada de CAFAM, Telesecundaria, Aceleración del aprendizaje y el Servicio de Educación Rural. Sin embargo, los interrogantes por la calidad de dichos programas no han sido evaluados a profundidad, tampoco se puede hacer evidente la pregunta por la población campesina como benefactora de los programas o, por lo menos, si se ha pensado para ellos.

Estas formas de cobertura que, desde la primera mitad del siglo Xx, se propusieron para zanjar la brecha existente en términos educativos, ya estaban permeados, como lo destaca Sandoval, por ser el formato de una "educación en las zonas rurales configurada como una institución que integra y transmite los valores y conceptos asociados a la idea de región, de nación y de pensamiento científico" (1996, p. 13). De esta manera, y

desde el punto de vista de la instrucción como de la formación, la escuela rural solo [se considera] por su ubicación física [como rural]. Sus contenidos, su método y la formación del maestro, visto aquí como el agente socializador son [netamente] urbanos.

(Sandoval, 1996, p. 15) 
En el marco de una escuela que forma en "masa" donde las desigualdades se refuerzan a la hora de aprender contenidos que luego no se ven reflejados en utilidad para la vida, más allá de la obtención de títulos que no tienen valor de uso, ni valor de cambio, la escuela que se interroga por una educación justa debería preocuparse por la formación que propone, lo que nos recuerda la dimensión ofrecida por Berstein (1972), una educación compensatoria que se ajusta al déficit como elemento para resolverlo. Esta tensión cobra igualmente su valor en la vida y educación rural con sus ritmos y sus propios contextos.

En consecuencia, las propuestas educativas rurales deben generar: 1) autonomía para adaptar las necesidades de cada entorno y las demandas de las escuelas de cada municipio y de sus estudiantes, padres de familia y comunidad; 2) una actitud que permita mantener el principio de elección como individuos; no para fijar posturas en contra de los diversos modelos educativos, sino para fundar una nueva propuesta escolar que pueda leer más allá del debilitado sistema escolar vigente. En este sentido, Candela (1995) analiza la profusa distancia que existe entre currículo propuesto y currículo real o vivido, "construido en la práctica de la educación"; allí se resalta la intervención que los docentes hacen sobre los conocimientos transmitidos y la disposición activa o pasiva de los estudiantes. De ellos proviene la relevancia de analizar las formas sociales de reproducción del conocimiento, sobre lo que infiere que el conocimiento se construye en el discurso, en la cotidianidad; en este punto hace fuerte hincapié en el "contexto", puesto que en él interactúa toda la vida cotidiana, el entorno, las ideas previas, los sujetos y por ende la cultura. Candela (1995) coincide con Bauman (2010), Escobar (2000), Giménez (2012) y Lefebvre (1974) al resaltar la relevancia de la existencia de significados, saberes y procesos de colectividades, en este caso el campesinado, que en el entramado de lo educativo, constituyen historias junto con sus territorios para configurar una educación que los involucre.

\section{Pensar una pedagogía de lo rural}

Quiero exponer de manera relacional parte del debate sobre la existencia de las organizaciones campesinas y el reconocimiento como sujeto de derechos del campesinado, dado que toda pedagogía pensada para lo rural debe ser, ante todo, como lo describe Borda (1978), una construcción que pueda evidenciar el problema de cómo investigar la realidad para transformarla por la praxis. Una razón es que la educación para lo rural, en el caso de Colombia, ha seguido un modelo totalmente occidental y tradicionalista, haciendo desaparecer toda práctica de saberes, costumbres del campesinado, así como lo enuncia Sandoval:
La escuela rural se transforma bajo el elemento de modernización, en cuanto encarna una visión de mundo y una forma de pensamiento modernizante e industrial [...] La nueva estructura de la sociedad y la idea de nación encierra los elementos de una nueva organización de la familia, la tecnología, la ciencia y en especial, la idea de comunidad que tiene y en la que vive el campesinado se ve transformada por la idea de nación que ingresa a la escuela rural, convirtiéndose [esta] en un vehículo homogeneizador de la cultura rural, al imponer a la forma de vida del campo, la visión de lo moderno a través de su modo de pensamiento científico-racional del cual es portadora la institución escolar. (1996, p. 372)

Por ello, antes de abordar un deber ser de la pedagogía rural, es necesario identificar esa realidad histórica del campesinado y los pobladores rurales avasallados por profundas inequidades, como base de la investigación-acción que Borda (1978) delegó.

Otra razón es la existencia de una población con procesos organizacionales, sistemas simbólicos y prácticas culturales inacabadas. Además, como lo describe Zemelman (1987), desde las voces de la ecología, la agricultura y la economía, los saberes y las prácticas campesinas han sopesado el valor referencial en el sentido de pérdida de identidad, de proyecto de sociedad. Así las cosas, considero que el tipo de pedagogía rural y su discurso debe ser abordado y construido con el fluir de esas dos consideraciones.

Solo en septiembre del 2018, la Organización de las Naciones Unidas (ONU), bajo los principios de dignidad y el valor inherente de los derechos de igualdad, reconoció los derechos de los campesinos y de otras personas que trabajan en la ruralidad. Es de gran valía esta declaración; empero, que Colombia se abstuviera de votar a favor, es un reto aún mayor para continuar exigiendo su reconocimiento tanto en las dinámicas de la vida cotidiana, como en los escenarios propios de la reflexión y la construcción de pensamiento, como lo es la academia. Es posible que las vinculaciones que han integrado diferentes entidades como la ONU o el PNUD con los términos de "desarrollo humano integral" aludan más al sentido de un procedimiento, como lo describe Walsh, en

la necesidad de la inclusión —de individuos de los grupos históricamente excluidos- como mecanismo para adelantar la cohesión social. Tal perspectiva se evidencia en los recientes cambios en la política de la Unesco, ahora dirigida a gestionar la diversidad para que no sea fuente de amenaza e inseguridad. (2010, p. 84) 
Ese esfuerzo, enuncia Walsh (2010), promueve imaginarios que con el discurso étnico e integrador benefician políticas económicas compatibles con el mercado. En ese orden de ideas, los efectos de la globalización contribuyen a la desaparición de las organizaciones campesinas, y pareciera ser uno de sus más destacados efectos; no obstante, las acciones de reivindicación para el campesinado siguen siendo adelantadas por los sectores campesinos. El caso más reciente destaca la acción de tutela para el reconocimiento de la categoría de campesino como sujeto de derechos, proceso que también se deriva del documento técnico elaborado por el Instituto Colombiano de Antropología e Historia (ICANH) en el 2017 sobre el concepto de campesinado. De allí se colige la existencia como movimiento dado que el ICANH

Emite el presente concepto en cumplimiento de lo acordado en el mes de julio de 2016 en la "Reunión de seguimiento y estado de avances de las acciones emprendidas por el gobierno nacional, en el marco de la Mesa Campesina del Cauca, CIMA - PUPSoc/ interlocución y negociación interministerial". En el debate sobre la inclusión del campesinado dentro de las preguntas del siguiente Censo Poblacional, se consideró que se requería un concepto técnico sobre campesino, que fuera la base para el trabajo que será desarrollado por el DANE. (2017, p. 1)

Se evidencia, por tanto, que las organizaciones campesinas mantienen una tensión constante por el no reconocimiento de su existencia en el mundo rural, los problemas agrarios, los conflictos por la tierra, la informalidad laboral, el desarrollo y el uso de la tierra. Estas situaciones, desde una lectura de la relación de productiva del campo, se ubican como uno de los hechos trascendentales de la productividad del país. En palabras de Suescún:

El problema agrario y los conflictos históricos del espacio rural colombiano comparten un núcleo común: la distribución de la tierra. Diversos estudios en diferentes etapas de la historia de Colombia y con distintos enfoques teóricos han hecho referencia a la persistencia e inercia a la concentración de la tierra. (2013, p. 655)

Este tipo de expresiones y reivindicaciones sugieren una postura diferente a la tradicional forma de reivindicación social por parte del campesinado. Siguiendo a Mondragón (s.f.), las luchas del campesinado que se gestaron a comienzos del siglo xx dieron origen a las "primeras organizaciones estables. Sus propuestas eran al principio meramente locales. Por ejemplo, en Viotá en 1934 los arrendatarios presentaron un pliego en el que simplemente proponían que se les dejara sembrar libremente café y establecer trapiches en las haciendas" (p. 1). Este tipo de solicitudes fueron creciendo en otros lugares del país como forma de movimiento campesino, constituyéndose en juntas, asambleas locales o habitando territorios baldíos.

En Colombia han confluido diferentes formas de violencia, ejercidas como medio para la consecución de cambios sociales. Esta situación ha variado en cada década; por ejemplo el control social, y la imposición económica, política y cultural han sido parte de dicho proceso. Esta situación ubica a los campesinos colombianos en una relación de desigualdad, visión de pobreza y atraso que, como lo menciona Barkin,

Justifican las políticas que después amenazan la propia existencia de los grupos sociales tradicionales y de sus sistemas productivos. Su incapacidad de adaptarse es evidencia que refuerza la idea de que estos grupos son la causa del atraso social y económico de las áreas rurales. Aún en las sociedades más modernas, "culpar a la víctima" de su propia situación y de su falta de progreso colectivo es un fenómeno bastante común. (1998, p. 3)

Esta lectura de la población rural, y en especial al campesinado como "pobres", es equivocada, dado que lo que se debe admitir es la negligencia y la débil capacidad para asignar recursos de las políticas públicas rurales. Así, la crisis de la ruralidad, como lo menciona Pérez (2001), se ve afectada por múltiples causas, en especial, las asociadas con el modelo de crecimiento económico, hecho que ejerce distintos tipos de presiones para la vida cotidiana de las familias campesinas. Empero, los procesos organizativos vienen a tomar relevancia para subsanar, mantener y construir otras formas de existir en sus territorios.

Por tanto, un grueso de la población rural ha encontrado en la idea del "movimiento social" una forma de resistir al distanciamiento ejercido por el Estado; para este caso, la figura de la organización campesina; en el presente documento no se aborde el recorrido de la presencia o momento de la configuración del movimiento campesino, pero sí, de alguna manera, sus impactos, tensiones y correlaciones en la sociedad colombiana, y en especial, la educación rural, han devenido de dicha configuración.

Existen otras dimensiones para asociar las dinámicas de los campesinos como sujetos. Una de ellas corresponde con la construcción de su identidad; la cual se expresa en sus narraciones o como actividad agrícola, al respecto, Vázquez, Ortiz, Zárate y Carranza (2013) mencionan que "existe un desconocimiento sobre cómo se identifican los campesinos" y proponen que una manera de conocerlo es mediante sus propias narraciones o discurso. Según Gergen, 
El discurso es el medio por el cual los individuos se hacen inteligibles. Al identificarse (con otros y consigo mismo), y escribirse en un tiempo y en un espacio de terminado; así crea un discurso acerca de sí mismo, producto de sus intercambios sociales. Después de todo "se cuenta la vida como relatos, y se viven las relaciones con los otros de una forma narrativa. (1996, p. 32)

El entramado de identidad suscribe por tanto una postura fundamental de lo organizativo, toda vez que el campesinado pasa por la anulación cultural. Allí la identidad, al igual que las actuales reivindicaciones como sujetos de derechos y como individuo, se puede considerar que es un producto social, ya que, como lo recalcan Vázquez et ál.:

Definirla no es algo simple; sin embargo, algunas características que permiten conceptualizar tal término con mayor precisión requieren considerar que: a) la identidad es compuesta: cada cultura o subcultura transportan valores e indicadores de acciones, de pensamientos y sentimientos; b) es dinámica: los comportamientos, ideas y sentimientos cambian según las transformaciones del contexto familiar, institucional y social en el cual se vive; y c) la identidad es dialéctica: su construcción no es un trabajo solitario e individual, requiere de la presencia de otros individuos. (2013, p. 3)

El centro de los cambios en la vida rural, han variado, por ello, resulta vital construir una lectura más ahincada de carácter nacional sobre las dinámicas de los saberes campesinos y su involucramiento en los procesos educativos para la ruralidad; ya que no solo se trata de explorar formas del hacer, del sentir y del pensar de los campesinos, sino que es imperativo afianzar con pertinencia argumentos para definir con mayor claridad aquello a lo que se denomina "ruralidad". Conceptos más precisos pueden servir para formular políticas adecuadas y pertinentes para la vida en el campo. Repensar esta dimensión, desde una perspectiva crítica, en la manera como a lo largo de la historia se han visto y han sido tratadas las personas que habitan las zonas rurales -especialmente las y los campesinos-, es abonar la lectura y la reflexión de algo inacabado. Allí, el papel de la educación que se imparte, con todas las potencialidades sociales, económicas, socializadoras y culturales que integran dichas poblaciones, es de vital importancia a la hora de pensar un mundo que puede cambiar y que se transforma en el bien común del campesinado.

\section{Devenir e identidad del campesinado}

Si bien las categorías identidad campesina y pedagogía rural son riesgosas, también lo puede ser la inexistencia de un modelo de educación que dé cuenta de las prácticas y los saberes campesinos. Esta limitación se puede rastrear en los lineamientos institucionalizados por el Ministerio de Educación en su línea del Proyecto Educativo Rural (PER), emprendido en el 2001 ( $\sin$ olvidar que dichas estrategias se han configurado desde 1947), como proceso de ampliación en cobertura educativa, no solo para el país, sino especialmente para lo rural, $\mathrm{y}$, a pesar de ello, no parece diferenciar, nombrar o reconocer a dicha población. La diversidad, como menciona Hall, "puede adoptar modalidades cerradas de cultura y de comunidad, negándose a comprometerse con los peliagudos problemas que provoca intentar vivir en la diferencia" (1993, p. 349). Al unísono, Escobar nos recuerda que "los lugares pueden ser olvidados, lo que significa su decadencia y deterioro; las gentes y el trabajo son fragmentados en el espacio de los lugares, en la medida en que los lugares son desconectados entre sí" (2000, p. 83). Esto no es más que la negación e invalidación de la existencia de una población. En palabras de Bauman,

La idea de "identidad" nació de la crisis de pertenencia y del esfuerzo que desencadenó para salvar el abismo existente entre el "debería" y el "es", para elevar la realidad a los modelos establecidos que la idea establecía, para rehacer la realidad a imagen y semejanza de la idea. (2010, p. 49)

Parafraseando a Bauman (2010), y para el caso del campesinado, la identidad es el reto para construir, y no la tarea ya acabada. Percibir las dinámicas de movilización de las organizaciones campesinas va más allá de lo etnográfico; implica entender la vida social a partir de las lógicas que construyen los sujetos sociales. Esto nos acerca a Hall (1993) cuando describe cómo representaciones de los procesos [en comunidad] van más allá del sentido y se afincan en el conocimiento de los contextos. Lo que en palabras de Borda (1978) nos ubica en el dilema de combinar lo vivencial con lo racional como un verdadero problema ontológico que no podemos eludir, en eso que él mismo denomina la angustiosa idea de investigar sin intención, sin un sentido, por el hecho de comprender la realidad, pero no poder ejercer cambios en ella, $y$ justamente ese debe ser el horizonte de sentido de la pedagogía rural. 
Las apreciaciones de De Sousa Santos ayudan a interiorizar los términos de la reivindicación, que muchas veces pareciera estar en contra de las postulaciones cientificistas, por no reconocer a poblaciones históricamente excluidas; más bien, es el uso contrahegemónico que

consiste, por un lado, en explorar la pluralidad interna de la ciencia, esto es, prácticas científicas alternativas que han sido hechas visibles por epistemología feministas y poscoloniales, y, por otro lado, en promover la interacción e interdependencia entre conocimientos científicos y no científicos.

(2010, pp. 52-53)

En ese sentido, la educación rural que reconoce al campesinado debe examinar la evidencia de sus alcances, límites e inserción en la comunidad. Esto implica conocer los contextos y saberes sobre la vida rural y el campesinado con relación a la educación de su entorno, de lo que Escobar colige una

defensa del lugar sin naturalizarlo, feminizarlo o hacerlo esencial, una defensa en la que el lugar no se convierte en la fuente trivial de procesos o fuerzas regresivas. Si uno ha de desplazar el tiempo y el espacio del lugar central que han ocupado en las ciencias físicas y sociales modernas — quizás incluso contando con las metáforas de las nuevas ciencias que resaltan las redes, la complejidad, la autopoesis, etc., conceptos éstos que no vinculan tanto al espacio y al tiempo- ¿es posible hacer eso sin reificar la permanencia, la presencia, la atadura, la corporeidad y similares? (2000, p. 69)

En ese proceso de la reivindicación del campesinado anida tanto lo decolonial, como lo asume De Sousa Santos (2010), como la idea de Lefebvre (1974) al enunciar que el espacio existe porque es donde habita el campesinado, y allí se anuda la pregunta por su proceso histórico. En medio de las transformaciones que les toca vivir, es válido preguntar si es posible una propuesta de educación rural que integre esos saberes campesinos, su identidad campesina y una pedagogía de lo rural o si, por el contrario, la educación que reciben en medio de los ajustes globales y mercantiles es suficiente para responder a sus necesidades y ritmos de vida.

En la dinámica del PER, el MEN carece de logísticas, así como de recursos económicos, didácticos y pedagógicos para proponer un modelo educativo diferente al productivo-economicista y empresarial que se edifica en el campo. En el 2012, el Ministerio publicó el manual para la formulación y ejecución de planes de educación rural, con el ánimo de alentar un tipo de educación rural diferencial; empero, sus disposiciones no superan el hecho agrario del campo como única forma del saber pedagógico — casi que es una burda forma de formar empleados agrarios-, y adolecen de una reducida y casi nula elaboración pedagógica que, como lo describe Núñez,

Otorga protagonismo a los sujetos locales como guardianes de un rico entramado de saberes salvaguardados, hibridados, sustituidos y emergentes (Núñez, 2003). Desde la subjetividad de los actores locales deben ser reconstruidos los patrimonios intangibles para obtener prácticas sociales apegadas a los procesos cognoscitivos propios de aprendizaje y enseñanza. (2006, p. 146)

Esto coincide con la idea de asumir una postura crítica sobre la manera como se ha venido haciendo investigación educativa, en especial en los países del "tercer mundo" donde es necesario tomar distancia y consciencia de los riesgos que puede ocasionar el abandono de lo conocido, y entender lo que se enseña en la escuela como lo único aprobado por la ciencia moderna. En este sentido, son pocos los trabajos que abordan una tensión cercana al tema sobre saberes campesinos en medio de la educación rural en Colombia, si bien existe una amplia caracterización de la educación rural. Por ejemplo, investigaciones como las desarrolladas por Perfetti (2003) que, desde la institucionalidad logran esbozar la condición crítica de los pobladores rurales y la situación de la escuela en términos económicos, de infraestructura y "calidad" educativa, no indagan por los currículos o las pedagogías, menos aún, por los saberes campesinos.

Algo similar ocurre con el trabajo de Sandra Milena Londoño y Javier Mauricio Mejía (2010), quienes destacan el programa de Acción Cultural Popular (ACPO) como uno de los más importantes para Colombia en materia de cubrimiento educativo. Este programa a cargo del clero, que para los años cincuenta estaba muy decaído, tiene un fuerte enfoque hacia la población campesina y el interés por mejorar y transformar sus condiciones de vida y educación. Este programa transmitido por Radio Sutatenza representa un ícono en la utilización de los medios de comunicación al servicio educativo, pero, persiste la idea de reproducir un modelo educativo nacional sin distinción de sector o población.

Por otro lado, se encuentra la experiencia de la "Universidad Campesina", que nace como una propuesta de las Comunidades de Paz en San José de Apartadó en 1997 y como alternativa a la cruda violencia a la que se veían abocados en el departamento de Urabá. Allí se colige una puesta en marcha de prácticas de saberes propios de la comunidad campesina para solventar las necesidades de comida, vivienda y siembra, derivadas de la guerra, donde la escuela 
y el comercio desaparecieron. Esta dinámica se ha tratado de reproducir en el departamento del Cauca por medio de sesiones itinerantes bajo la tutela de un equipo dinamizador que define con la comunidad el tiempo y el ritmo de trabajo, pero ha tenido poco desarrollo e implementación; estos hechos están a la base de haber reconocido un proceso capitalista en la educación como bien lo muestra Mejía:

la mirada sobre la escuela de corte reproduccioncita de tipo laboral no alcanza a entender cómo se ha modificado el papel de esta en el capitalismo globalizado y neoliberal, llevando a muchos a mantener una crítica desde las miradas del pasado que no da cuenta suficientemente de las transformaciones en marcha. Por ello, hablamos también de la necesidad de reinventar la crítica, cuestionando la forma y el contenido de la crítica del pasado. (2006, p. 35)

Por su parte, la Escuela Nueva ha servido de corolario para representar uno de los modelos más destacados de cubrimiento escolar en el país, a pesar de que la versión que se implementó desde los años ochenta en Colombia con el mismo nombre no representa las dimensiones sociales, culturales, políticas y religiosas de las ideas escolanovistas con las que ingresó en la primera década del siglo xx. Además, esta Escuela Activa representó "la creación de todo el sistema educativo nacional, como una de las estrategias que ayudaría a la consolidación del Estado-nación" (Herrera, 1999, p. 63).

Esta disertación sobre la educación rural deja por fuera la existencia de un proyecto de inserción de prácticas campesinas o rurales en los programas educativos. Por ello es imperativo decir que lo que ha existido en Colombia es un modelo educativo nacional unificado, donde la pedagogía, los currículos y las metodologías presentes y desarrolladas en la educación rural son exactamente equiparables al modelo urbano de educación, así se mantenga desde el 2001 el Programa de Educación Rural del Ministerio de Educación.

Hacemos referencia a la ruralidad y a la necesidad de un modelo educativo acorde a la vida del campo, porque en la ruralidad el conocimiento se construye colectivamente, es un proceso de aprendizaje en el que el estudiante participa de manera activa y los docentes se hacen partícipes en la construcción de saberes como orientadores y guías en relación permanente con su entorno. Esto implica romper en gran medida con la forma tradicional del salón y el tablero; no siempre ni todos los días se aprende encerrado en cuatro paredes - a lo que se le denomina salón de clase- Para los niños y niñas de la ruralidad es importante el contacto con el entorno; allí están los saberes de sus padres, de su cultura, de su forma de ver y entender el mundo, y la educación debe ofrecer esa posibilidad. Sin embargo, como afirma Zambrano,

Las dificultades de la sociedad colombiana para proteger la vida humana y para garantizar los elementos mínimos de un orden social abierto y democrático no pueden ser registradas por una política educativa que insiste en el disciplinamiento de las instituciones educativas. Las preguntas por los sentidos y las implicaciones del esfuerzo educativo de las sociedades deben ubicarse en el centro de la agenda de una sociología educativa acorde con las particularidades colombianas. (2005, p. 146)

Por ello, hablar de una pedagogía de la ruralidad, que reconozca la complejidad de lo local y la necesidad de lo nacional, es imperativo, así como lo es una pedagogía que instaure un diálogo de saberes con la comunidad y a su vez lo vuelva currículo; pero, sin olvidar, como lo dice Perrenoud, que "ninguna renovación curricular creará por sí misma las condiciones para la igualdad de los conocimientos adquiridos" (2010, p. 114), ni mucho menos que certificar conocimiento es garantía de éxito social.

\section{Un saber desigual}

El conocimiento y sus diversos campos han sido organizados en "occidente bajo los modelos de las ciencias modernas". Según Canclini (2004), en Latinoamérica, las prácticas y los saberes de la población indígena, su medicina tradicional, sus construcciones artesanales y las formas organizativas con las que usan el conocimiento coexisten con las ciencias.

Pese al reconocimiento desigual que reciben los saberes científicos y los tradicionales, las tendencias evolucionistas que tienden a descalificar a las culturas indígenas, los saberes autóctonos siguen siendo utilizados por vastos sectores como recursos para la salud, para el trabajo campesino y la educación cotidiana. (Canclini, 2004, pp. 182-183)

La idea de considerar la desigualdad como única dimensión de la exclusión evita formular la relación social que derivada del enfoque modernista, de entender los procesos de integración económica como soporte de gran parte de los procesos de globalización y modernidad. Como lo describe Castro, "una modernidad que funciona como una máquina generadora de alteridades que, en nombre de la razón y el humanismo, excluye de su imaginario la hibridez, la multiplicidad, la ambigüedad y la contingencia de las formas de vida concretas" (2000, p. 145). Al respecto Lander dice que 
La conquista ibérica del continente americano es el momento fundante de los dos procesos que articuladamente conforman la historia posterior: la modernidad y la organización colonial del mundo. Con el inicio del colonialismo en América comienza —no solo la organización colonial del mundosino simultáneamente la constitución colonial de los saberes, de los lenguajes, de la memoria y del imaginario. (2011, p. 16)

De la misma manera, cuando se aboca a la globalización con vínculo a la educación, concordamos con Bonal al decir que "son por lo tanto dos caras de una misma moneda que explican la paradoja de la necesidad y la insuficiencia de la educación" (2005, p. 86), al no resolver la brecha de pobreza $y$, al contrario, profundizar la desigualdad. Como el mismo Zambrano ratifica para el caso de Colombia, "El modelo educativo no logra corregir el marginamiento estructural que sufren miles de jóvenes del país" (2005, p. 147), quizá, porque en medio de los desarrollos educativos persiste lo que Bernstein (1993) denominó "enmarcación" y se regulan las prácticas educativas e institucionales al punto de mercantilizar el conocimiento dentro de un sistema colonial, tal como Quijano nos propone:

\footnotetext{
Es en primer término, la culminación de un proceso que comenzó con la constitución de América y la del capitalismo colonial/moderno y eurocentrado como un nuevo patrón de poder mundial. Uno de los ejes fundamentales de ese patrón de poder es la clasificación social de la población mundial sobre la idea de raza, una construcción mental que expresa la experiencia básica de la dominación colonial, que, desde entonces, permea las dimensiones más importantes del poder mundial, incluyendo su racionalidad específica, el eurocentrismo. Además, considera que esa construcción fue el primer "espacio/tiempo de un nuevo patrón de poder de vocación mundial y, de ese modo, la primera identidad de la modernidad. (2000, pp. 201-202)
}

Esta es la razón, dice él, por la cual se instaura la formación de relaciones sociales que funda la idea que produjo en América las identidades sociales históricamente nuevas, indios, negros y mestizos, y redefinió otras. Por ello, es meritorio considerar la sugerencia de Wallerstein (1996) de rechazar la distinción ontológica entre los seres humanos y la naturaleza, dado que los rasgos propios - como considera Quijano (2000) cuando habla de raza-, en su sentido moderno, no tienen historia conocida antes de América. Quizás se originó como relato de las diferencias fenotípicas entre conquistadores y conquistados, pero lo que importa es que muy pronto fue construida como referencia a supuestas estructuras biológicas diferenciales entre ellos.

No obstante, queda la noción de que "un pueblo que no tiene conciencia de su pasado, no tiene dominio sobre su futuro. Evidentemente todo pueblo, aunque no tenga conciencia, tiene un pasado, en ese mismo sentido tiene futuro" (Dussel, 1973, p. 34). La idea que se debe resaltar es que los seres humanos no son únicamente el producto de la distinción entre sociedad y naturaleza, como lo considera Appadurai (1996); la cultura no es un sustantivo, como si fuera algún tipo de objeto o cosa, sino un adjetivo, esto es "el aspecto más valioso del concepto de cultura es el concepto de diferencia, una propiedad contrastiva -más que una propiedad sustantiva-" (Appadurai, 1996, p. 14); dicho de otro modo, la cultura no debe considerarse una esencia o algo que porta en sí cada grupo, sino como el "subconjunto de diferencias que fueron seleccionadas y movilizadas con el objetivo de articular las fronteras de la diferencia" (Appadurai citado por Canclini, 2004, p. 39).

En este sentido, Zemelman "se plantea una ampliación en la relación de conocimiento de modo que sea congruente en la incorporación del sujeto en sus circunstancias, lo que obliga a concebir al conocimiento como parte de una relación más incluyente" (2010, p. 6). Esta tendencia en la construcción constante de conocimiento se opone a la manera como solían ser las ciencias sociales en los siglos XVII y XVIII, las cuales:

\section{Se hallan sostenidas por un imaginario colonial de carácter ideológico. Conceptos binarios tales como barbarie y civilización, tradición y modernidad, comunidad y sociedad, mito y ciencia, infancia y madurez, solidaridad orgánica y solidaridad mecá- nica, pobreza y desarrollo, entre otros muchos, han permeado por completo los modelos analíticos de las ciencias sociales. (Castro, 2000, p. 93)}

El imaginario del progreso según el cual todas las sociedades evolucionan en el tiempo bajo leyes universales inherentes a la naturaleza o al espíritu humano aparece como un producto ideológico construido desde el dispositivo de poder moderno/ colonial. Las ciencias sociales funcionan estructuralmente como un "aparato ideológico" que, de puertas para adentro, legitimaba la exclusión y el disciplinamiento de aquellas personas que no se ajustaban a los perfiles de subjetividad que necesitaba el Estado para implementar sus políticas de modernización. De puertas para afuera, en cambio, las ciencias sociales legitimaban la división internacional del trabajo y la desigualdad de los términos de intercambio y comercio entre el centro y la periferia, es decir, los grandes 
beneficios sociales y económicos que las potencias europeas estaban obteniendo del dominio sobre sus colonias (Castro, 2000).

Las dinámicas de exclusión coadyuvan a establecer formas de inclusión. Ahora bien, Moreno se interroga por el tipo de proceso que subyace en dicho proceso: “¿de qué inclusión y de qué exclusión se trata? ¿En qué se está incluido y de qué se está excluido?" (2000, p. 164). Sin embargo, de lo que se trata es de poder ubicar las dimensiones de la vida cotidiana sobre los cuales se han situado y enfrentado las disputas por el saber y la legitimidad. Al respecto Dussel comenta:

\begin{abstract}
Ahora se trata de "situar" a todas las culturas que inevitablemente se enfrentan hoy en todos los niveles de la vida cotidiana, de la comunicación, la educación, la investigación, las políticas de expansión o de resistencia cultural o hasta militar. Los sistemas culturales, acuñados durante milenios pueden despedazarse en decenios, o desarrollarse por el enfrentamiento con otras culturas. Ninguna cultura tiene asegurada de antemano la sobrevivencia. Todo esto se ha incrementado hoy, siendo un momento crucial en la historia de las culturas del planeta. (2005, p. 12)
\end{abstract}

De esta manera, ubicar el modelo de educación colombiano y su proceso de constitución entraña esas dinámicas de inclusión/exclusión propia del proceso modernizador. Siguiendo a Canclini, "las naciones tenían culturas más o menos autocontenidas, con ejes ideológicos definidos y perseverantes, que regían la mayor parte de la organización económica y las costumbres cotidianas. Se creía saber qué significaba ser francés, ruso o mexicano" (2004, p. 16). De ahí que la primera cuestión que se tiene que plantear como eje ordenador del pensamiento es lo que concierne al orden, en cuanto este conforma un mecanismo constitutivo de subjetividades mediante sus múltiples recursos al influir sobre el sistema de necesidades de los colectivos sociales. De hecho, al considerar la existencia de un sujeto situado en relaciones múltiples, como describe Zemelman:

Conforman el espacio que los determina en la naturaleza de su movimiento, que se traduce en primer lugar, en el surgimiento de la necesidad de ocupar un espacio en el que tiene lugar el reconocimiento a pertenencias colectivas, lo que se acompaña de la conformación de una subjetividad social particular. (2010, p. 2)

Valorar las consideraciones que Lefebvre (1974) hace sobre las prácticas espaciales como reguladoras de la vida es crucial, además de aclarar que el espacio no tiene ningún poder "en sí" y las contradicciones del espacio no son determinadas por él, como tal. Son en sí, contradicciones de la sociedad (entre esto y aquello en la sociedad, por ejemplo, entre las fuerzas productivas y las relaciones de producción); "ellas se actualizan en el espacio, a nivel del espacio, engendrando las contradicciones del espacio" (Lefebvre, 1974, p. 268). Aquí la historia de la educación en Colombia parece discriminar positivamente su población rural.

Cuando se tiene 6, 9 o 12 años de edad, luego de madrugar a las cuatro de la mañana, alistarse para caminar dos o tres horas por senderos enlodados para llegar a la escuela, bordeando montañas o en llanuras lejanas, sin la ruta escolar, quizá sin internet, sin bibliotecas, con muchos lugares sin electricidad, o esperando que sean las tres de la tarde para aprovechar las cinco horas de energía que proviene de la planta eléctrica, con los tañidos de la guerra aún cerca, y luego regresar a la casa para ayudar en las labores de la finca, es imaginable que ese modelo educativo debe considerar el contexto social, histórico y poblacional en el que se instaura la escuela para formular sus contenidos curriculares. Como lo enuncia Edelman, al abordar la categoría de campesino, existen unas "complejas realidades de los pueblos emigrantes, desterritorializados, con diversas ocupaciones y que dependen de diversas corrientes de ingresos" (1998, p. 279), pero que se hace peligroso cuando reclama mayor bienestar social porque los campesinos saben que la transformación de la sociedad supone la gestión colectiva de su propio espacio como población.

\section{Conclusiones}

Las iniciativas de las comunidades rurales nacen de la necesidad de proponer y reclamar el derecho a ser tratadas diferencialmente. Estos sectores comparten un mismo territorio con pueblos originarios (población indígena) y con poblaciones que tienen otras formas de vincularse con la tierra y el mundo (campesinos), pero necesitan y requieren estrategias donde puedan relacionarse entre las diferentes poblaciones rurales, donde nadie sienta amenazada la existencia de su cultura o cosmovisión, sino, por el contrario, puedan ser un complemento entre ellas; de manera tal que las distintas formas de ver y articularse con el mundo puedan estar presentes en el espacio escolar, donde la conformación de un currículo educativo nacional para la educación básica, media y de la primera infancia vincule la idea de una pedagogía rural. En ese sentido, las siguientes consideraciones son apenas algunos vórtices que a la distancia aparecen como correlato sintético: 
- Las particularidades territoriales y culturales en las que viven los campesinos, a quienes el Estado debe garantizar una educación relacionada con prácticas sociales, visiones de mundo - las cuales históricamente les ha permitido sobrevivir en sus sistemas sociales-, deben ser visibles en una educación rural más pertinente que preserve la identidad, el arraigo a su tierra, la sostenibilidad ambiental y su propio autorreconocimiento.

- En la diversidad de regiones del país donde habitan los campesinos, existen particularidades ambientales, geográficas, tradicionales, históricas, políticas y culturales, que ofrecen razones y aportes válidos para pensar en la construcción de currículos locales adaptados a los espacios donde ellos habitan. Esto presupone una pedagogía de formación y preparación especial para los docentes, para que puedan diferenciar entre lo tradicional y lo oportuno para el contexto donde desarrolla su proceso de formación.

- A la educación rural se le debe asignar valor y estatus académico cuando de saberes locales (campesinos) se trata, similares a los otorgados a los saberes universales, suscritos a la usanza del valor del patrimonio material e inmaterial, y de esta manera afincar su identidad.

- Se debe facilitar la construcción de una herramienta teórica y metodológica sobre los modos en que se produce el aprendizaje campesino para acercarlo a los procesos de enseñanza-aprendizaje, que puedan facilitar su implementación educativa y puedan, además, servir para aplicar o mejorar las iniciativas en otros contextos donde los currículos educativos son estrictamente homogéneos.

- Los diseños de los currículos deben tener la participación efectiva y activa de la comunidad, para que de esa manera puedan tomar postura y acción sobre lo que desean aprender sus miembros. Algunas de las preguntas que debería tener sus programas y proyectos escolares son ¿para qué deben estudiar?, ¿cómo deben recibir su educación?, ¿cuáles son los espacios escolares adecuados?, ¿cómo debe ser el calendario escolar y los contenidos más relevantes?, ¿cuál es el perfil que debe tener el docente rural y el tipo de estudiante que desean formar?

No se trata de aventurar un idealismo fundado, sino de reiterar las decisiones consensuadas entre los actores educativos y los actores comunitarios que las instituciones garantes de los derechos educativos tanto desconocen. Reivindicar lo identitario tiene que ver con la inexistencia de una definición concisa sobre lo que significa ruralidad. Cuando hablamos de ella, hacemos referencia a hábitos relevantes de la población rural, pero la reflexión queda abierta para seguir apuntando a un concepto más pertinente que sirva para formular políticas adecuadas y pertinentes para sus pobladores; el tema queda como algo inacabado, con todas las potencialidades sociales, económicas, socializadoras y culturales que integra la diversidad de poblaciones. Para ello, la educación y la pedagogía rural constituyen los mundos, ajustes y reivindicaciones de las poblaciones del mundo rural. La educación es rural, no porque se estudie en el campo, sino porque se integran sus saberes, la cosmovisión, la cultura y experiencias de la vida cotidiana de sus pobladores, y la relación con otras maneras del aprender, del hacer y del enseñar.

La educación rural y la vida en el campo están mediadas por relaciones de poder mercantil, sinónimo de desarrollo. Ello genera pérdida de identidad y de tradición cultural. La población campesina es la más afectada por dicho proceso, al punto que ni los habitantes que comparten el mismo territorio los valoran como sector excluido. Ante dichas deficiencias se hace imperativo tejer relaciones para afianzar con más ahínco argumentos de reconocimiento de la población campesina para que la educación sea pertinente al mundo rural; para ello, ha de fraguarse algo más que una reflexión. Este ejercicio no zanja en ningún momento el historial ya conocido de pobreza, desigualdad y distancia en ámbitos de conectividad y programas académicos de nuevas tecnologías aplicadas en la educación rural, por tanto, los discursos educativos que imperan y persisten en ello deben cambiar; pareciera más que el ajuste sigue siendo mantener niveles mínimos de conocimiento y acceso al mundo rural, por considerárselo aún en atraso y marginalidad.

\section{Referencias}

Arias, J. (2017). Problemas y retos de la educación rural colombiana. Educación y ciudad, (33), 53-62.

Appadurai, A. (1996). La modernidad desbordada. Dimensiones culturales de la globalización. Trilce.

Apple, M. W. (1979). Ideology and curriculum. Routledge.

Apple, M. W. (1997). Maestros y textos. Una economía política de las relaciones de clase y de sexo en la educación. Paidós.

Barkin, D. (1998). Riqueza y pobreza. Pobreza y desarrollo sostenible. Jus. 
Bernstein, B. (1972). Education cannot compensate for society [La Educación no puede suplir las fallas de la sociedad, trad. N. Conover]. https://nanopdf.com/download/ la-educacion-no-puede-suplir-las-fallas-de-la_pdf

Bernstein, B. (1993). La construcción social del discurso pedagógico (M. Díaz, trad.). El Griot.

Bauman, Z. (2010). Identidad. Conversaciones con Benedetto Vecchi. Losada.

Borda, F. (1978). El problema de cómo investigar la realidad para transformarla. Federación para el Análisis de la Realidad Colombiana. http://www.ts.ucr.ac.cr/binarios/pela/pl-000411.pdf

Bourdieu, P. (1998). Capital cultural, escuela y espacio social. Siglo XXI.

Bonal, X. (2005). Las políticas de las omisiones: globalización, educación y pobreza en América Latina. Revista colombiana de Sociología, 81-104.

Candela, A. (1995). Transformaciones del conocimiento científico en el aula. La escuela cotidiana. Fondo de Cultura Económica.

Canclini, G. (2004). Diferentes, desiguales y desconectados. Mapa de la interculturalidad. Gedisa.

Castro, S. (2000). Ciencias sociales, violencia epistémica y el problema de la inversión del otro. En E. Lander (comp.), La colonialidad del saber, eurocentrismo y ciencias sociales, perspectivas latinoamericanas (pp. 88-98). Clacso.

De Sousa Santos, B. (2010). Descolonizar el saber, reinventar el poder. Trilce.

Dussel, E. (1973). América Latina. Dependencia y liberación. Clacso.

Dussel, E. (2005). Transmodernidad e interculturalidad. Interpretación desde la filosofía de la liberación. UAM-Iz.

Escobar, A. (2000). El lugar de la naturaleza y la naturaleza del lugar: ¿globalización o postdesarrollo? En E. Lander (comp.), La colonialidad del saber: eurocentrismo y ciencias sociales. Perspectivas latinoamericanas (pp. 68-87). Clacso.

Edelman, M. (1998). El movimiento campesino transnacional de América central. Revista Mexicana de Sociología, 60(4), 277-319.

Gergen, K. J. 1996. Realidades y relaciones: aproximaciones a la construcción social. Paidós.

Giménez, G. (2012). La cultura como identidad y la identidad como cultura. Instituto de Investigaciones Sociales de la UNAM,. https://perio.unlp.edu.ar/teorias2/textos/ articulos/gimenez.pdf
Hall, S. (1993). Culture, Community, Nation. Cultural Studies, 7(3). https://doi.org/10.1080/09502389300490251

Hernández, R. (1993-1994). Teorías sobre el campesinado en América latina. Una evaluación crítica. Revista Chilena de Antropología, 12, 179-200.

Herrera, M., Pinilla, A. y Sauza, M. (2003). La identidad nacional en los textos escolares de ciencias sociales. Colombia: 1900-1950. Universidad Pedagógica Nacional.

Herrera, M. C. (1999). Modernización y Escuela Nueva en Colombia 1914-1951. Universidad Pedagógica Nacional.

Instituto Colombiano de Antropología e Historia. (2017). Estudio técnico sobre el concepto de campesino en Colombia. https://www.dejusticia.org/wp-content/ uploads/2017/11/Concepto-t\%C3\%A9cnico-del-Instituto-Colombiano-de-Antropolog\%C3\%ADa-e-Historia-ICANH.pdf

Lander, E. (comp.). (2011). La colonialidad del saber: eurocentrismo y ciencias sociales. Clacso.

Lefebvre, H. (1974). La construcción del espacio. Anthropos.

Londoño, M. y Mejía J. (2010). El discurso de una ética católica modernizada. El caso del programa Acción Cultural Popular. 1947-1958 [tesis de maestría, Universidad Pedagógica Nacional].

Machado, A. (2002). De la estructura agraria al sistema agroindustrial. Universidad Nacional de Colombia.

Martínez, A., Noguera, C. y Castro, O. (1994). Currículo y modernización. Cuatro décadas de educación en Colombia. Universidad Pedagógica Nacional.

Mejía, M. R. (2006). Educación(es) en la(s) globalización(es) I, entre el pensamiento crítico y la nueva crítica. Ediciones Desde Abajo.

Ministerio De Educación Nacional. (1995). Decreto 804 de 1995. Reglamentario del título III capítulo 3. de la ley 115 de educación para grupos étnicos. http:// www.alcaldiabogota.gov.co/sisjur/normas/Norma1. jsp?i=1377

Ministerio De Educación Nacional. (2001). Ley 715. https:// www.mineducacion.gov.co/1621/articles-86098_ archivo_pdf.pdf

Mondragón, H. (s.f). Expresiones del campesinado. https:// www.researchgate.net/profile/Hector_Mondragon3/ publication/326979386_EXPRESIONES_DEL_CAMPESINADO_Hector_Mondragon/links/5b6f373545851546c9fb73b3/EXPRESIONES-DEL-CAMPESINADO-Hector-Mondragon.pdf 
Moreno, A. (2000). Superar la exclusión, conquistar la equidad: reformas, políticas y capacidades en el ámbito social. En E. Lander (comp.), La colonialidad del saber: eurocentrismo y ciencias sociales. Perspectivas latinoamericanas (pp. 99-121). Clacso.

Núñez, J. (2006). La investigación educativa revalorizadora: una vía para preservar la diversidad cultural. Sinopsis Educativa, Revista Venezolana de Investigación, (2), 135-146.

Ortiz, S. (1979). Reflexiones sobre el concepto de la cultura campesina y los sistemas cognoscitivos campesinos. En Teodor Shanin (ed.), Campesinos y sociedades Campesinas. Fondo de Cultura Económica.

Organización de las Naciones Unidas. (2018). Declaración de los derechos de los campesinos y campesinas.

Pérez, E. (2001). "Hacia una nueva visión de lo rural". En Pensando el desarrollo rural (parte I). http://biblioteca. clacso.edu.ar/clacso/gt/20100929011414/2perez.pdf

Perfetti, M. (2003). Estudio sobre la educación para la población rural en Colombia. CRECE. http://www.redler.org/estudio educación población rural colombia.pdf

Perrenoud, P. (2010). Los ciclos de aprendizaje, un camino para combatir el fracaso escolar. Magisterio.

Programa de las Naciones Unidas para el Desarrollo (PNUD). (2011). Colombia rural. Razones para la esperanza. Informe Nacional de Desarrollo Humano. Bogotá. INDH-PUND.

Quiceno, H. (2003). Crónicas históricas de la educación en Colombia. Magisterio.

Quijano, A. (2000). Colonialidad del poder, eurocentrismo y américa latina. En E. Lander (comp.) La colonialidad del saber: eurocentrismo y ciencias sociales (pp. 122151). Clacso.

Rojas, A, y Castillo E. (2005). Educar a los otros. Estado, políticas educativas y diferencia cultural en Colombia. Editorial Universidad del Cauca.
Sandoval, R. (1996). Escuela y modernidad en Colombia. Tercer Mundo.

Suescún, C. A. (2013). La inercia de la estructura agraria en Colombia: determinantes recientes de la concentración de la tierra mediante un enfoque espacial. En Cuadernos de Economía, 32(61), 653-682.

Tadeu da Silva, T. (1997). Descolonizar el currículo: estrategas para una pedagogía crítica (dos o tres comentarios sobre el texto de Michael Apple). En Apple Michael; Silvia Tomaz; Tadeu de y Gentile Pablo (ed.) 1997, Cultura, política y currículo. Ensayos sobre la crisis de la educación pública (pp. 64-78). Losada.

Vázquez, A., Ortiz, E., Zárate, F. y Carranza, I. (2013). La construcción social de la identidad campesina en dos localidades del Municipio de Tlaxco. http://www. scielo.org.mx/scielo.php?script=sci_arttext\&pi$\mathrm{d}=\mathrm{S} 1870-54722013000100001$

Walsh, C. (2010). Interculturalidad crítica y educación intercultural. En J. Viaña, L. Tapia y C. Walsh (eds.), Construyendo interculturalidad crítica (pp. 75-96). Instituto Internacional de Integración del Convenio Andrés Bello.

Wallerstein, I. (1996). Abrir las ciencias sociales. Siglo XXI.

Zambrano, M. F. (2005). Políticas de calidad educativa en una sociedad neo-feudal. El caso de Colombia. Revista Colombiana de Sociología (25), 127- 148.

Zemelman, H. (1987). Uso crítico de la teoría: en torno a las funciones analíticas de la totalidad. Colegio de México.

Zemelman, H. (2010). Sujeto y subjetividad: la problemática de las alternativas como construcción posible. Polis, Revista de la Universidad Bolivariana, 9(27), 355-366. http://es.scribd.com/doc/213241313/ Zemelman-Hugo-Sujeto-y-Subjetividad 